\title{
SHOCK PROTECTION USING SOFT COATINGS AS SHOCK STOPS
}

\author{
Sang Won Yoon ${ }^{1}$, Sangwoo Lee ${ }^{1}$, Noel C. Perkins ${ }^{2}$, and Khalil Najafi ${ }^{1}$ \\ ${ }^{1}$ Center for Wireless Integrated Microsystems (WIMS) \\ ${ }^{2}$ Department of Mechanical Engineering \\ University of Michigan, Ann Arbor, MI, USA
}

\begin{abstract}
This paper reports the modeling, fabrication and testing of shock stops formed using soft material coatings. These shock stops provide outstanding shock protection by reducing both the impulse and impact force, and by absorbing the shock energy at each impact following the applied shock. Design tradeoffs are described based on the elastic model of the thin film coated on hard shock stops. Parylene is used as the coating material due to its conformal coverage, room-temperature deposition, and chemical stability. Shock tests were performed on silicon test devices consisting of a proof mass suspended using a flexible spring, some coated with a soft coating (Parylene) and some without any coatings. These tests show that a $3 \mu \mathrm{m}$-thick Parylene coating provides superior device survival rate (94\%) compared with conventional hard stops $(0 \%)$ for applied shock levels of up to $1300 \mathrm{~g}$.
\end{abstract}

\section{INTRODUCTION}

The performance and reliability of MEMS devices are easily impacted by external environmental disturbances including incident mechanical shocks. MEMS devices that utilize delicate mechanical structures are susceptible to these shock loads, which may ultimately damage the device or degrade its performances.

To minimize these adverse effects, two solutions are typically proposed. One solution ensures that the shock-induced stress remains less than the material fracture strength by adjusting the dimensions of the structure. The other solution employs hard wall shock stops to limit the travel of the device [1]. Both solutions are attractive, yet they also have shortcomings. The first method achieves improved shock resistance at the expense of device performance [2]. The second method only offers marginal protection since it can itself generate secondary sources of shock (e.g. subsequent impacts) that may result in fracture, debris, performance shifts, or continuous ringing of the device [1-3]. Effective shock protection technologies must limit these shortcomings as well as minimize device displacement.

To achieve these goals, we proposed two new shock stop concepts utilizing nonlinear springs and soft coatings [1,2]. The merits and demerits of each concept were analyzed using simulations and the design parameters needed to control the critical target shock amplitude were determined. These simulations revealed a significant reduction of impulse generated at $1^{\text {st }}$ impact (nonlinear spring: $>90 \%$, gold soft coating: $>40 \%$ ) and a substantial decrease in the number of collisions (gold soft coating: $>90 \%$ ) compared to conventional hard wall stops.

The fabrication and testing of the nonlinear spring shock stops integrated with two MEMS devices with different sensing mechanisms and made by different processes was presented in [3]. Shock tests using an impact hammer demonstrated that the nonlinear spring shock stops provide outstanding device survival rate $(83 \%)$ compared with hard stops $(8 \%)$. Also, device fracture mechanisms under the stress developed by the induced impact force were identified.
This paper reports on the modeling, fabrication and testing of soft coating shock stops using Parylene as the coating material.

\section{CONCEPT}

Figure 1 shows the schematic view of a conventional hard shock stop and the proposed soft coating shock stop. When the device mass impact a shock stop, an impact force is generated. This force can be reduced by either 1) increasing the contact time between the mass and the stop assuming the impulse stays constant, or 2) decreasing the impulse (i.e., decreasing the momentum change) assuming the contact time stays constant. The impulse is proportional to the change in proof mass velocity [3].

This goal is achieved by adding a soft coating shock stop. This concept utilizes a soft thin-film layer on an otherwise hard surface, and it relies both on the increased surface compliance and dissipation. The soft coating reduces the coefficient of restitution (COR) and thus reduces the impact force. The 'softer' coating dissipates energy during impact, and this serves to reduce both the number of impacts as well as the settling time following shock. Increasing the damping of the device mass is another means to minimize the setting time, but this method is limited by the vacuum environment required by many MEMS devices. Thus, especially in the case of vacuum packaged MEMS, absorbing the energy at the impact site becomes more attractive [2].
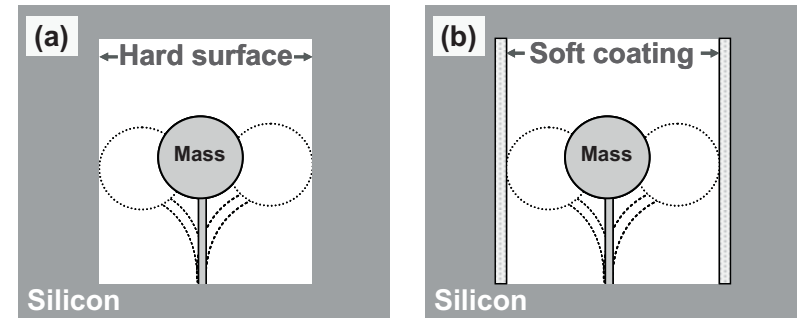

Figure 1. Schematics of shock stop designs

(a) Conventional hard shock stop, (b) Soft coating shock stop.

Soft coating and nonlinear spring shock stops have several similarities and differences. First, both concepts utilize compliant stops to reduce the impulse (and impact force). The soft coating stop however adds considerably more dissipation and hence it also reduces the number of impacts [2]. Second, these two shock protection concepts require different fabrication processes. For inplane shock protection, nonlinear spring stops can be readily fabricated with MEMS devices in a single step [3], but soft coating stops need additional steps due to the need for additional material deposition. For out-of-plane protection, soft coatings may be more convenient because it is difficult to fabricate released microsprings under a device. Finally, we can readily control our target shock amplitude for both concepts by simple design adjustments. The performance of nonlinear spring stops can be easily adjusted by changing the geometric and material properties (number, separation, stiffness) of shock springs [3]. The characteristics of soft coating stops are determined both by the coating material 
properties and thickness, and by the design of bumpers contacting the coated surface, as described in following sections.

\section{DESIGN}

Our previous analytical results demonstrated that gold ( $\mathrm{COR} \approx 0.22$ ) coatings reduce the initial impulse by $>40 \%$ and the number of collisions by $>90 \%$ as compared to a rigid stop [1, 2]. But these results were calculated by considering only the reduced COR; these results could be further improved by including the elastic properties of the coating materials. Moreover, these benefits are amplified by using flexible polymers because of their viscoelastic properties, smaller Young's modulus, higher energy absorption, and greater thickness.

$\mathrm{Yu}$ et al. reported simple but reliable models to analyze the elasticity of a thin film layer [4]. This work has been experimentally proven with minor corrections or referenced by many follow-up papers [5-7]. These models describe the deflection $(d)$ of a thin film due to a load $(p)$ applied by an indenter as a function of the indenter's shape and dimensions. The film is assumed to be either perfectly bonded to or frictionless overlaying on a semi-infinite substrate (Figure 2). The equations relating $p$ and $d$ are given as follows for different indenter shapes:

$$
\begin{aligned}
& \text { Flat-ended indenter (Figure 2-a): } p=\left(\zeta_{i} \cdot a\right) \cdot d \\
& \text { Conical indenter (Figure 2-b): } p=\left(\zeta_{i} \cdot \tan \alpha\right) \cdot d^{2} \\
& \text { Spherical indenter (Figure 2-c): } p=\left(\frac{2}{3} \zeta_{i} \cdot \sqrt{R}\right) \cdot d^{3 / 2}
\end{aligned}
$$

The definition of the other variables in these equations is shown in Figure 2. $\zeta_{\mathrm{i}}$ is defined to be $\zeta_{\mathrm{i}}=4 \mathrm{E}_{\mathrm{i}} /\left(1-v_{\mathrm{i}}\right)$ where $\mathrm{E}_{\mathrm{i}}$ is Young's modulus and $v_{\mathrm{i}}$ is Poisson's ratio. Note that a film's indentation is related to its contact area, not to its total surface area. This is observed in a similar manner both for purely elastic and elastic-plastic indentations, as reported in other papers [5,6]. When the film thickness approaches infinity, $E_{i}$ and $v_{i}$ approach those of the thin film material $(i=1)$. When the film thickness approaches zero, $\mathrm{E}_{\mathrm{i}}$ and $v_{\mathrm{i}}$ approach those of a hard substrate material $(\mathrm{i}=2)$. Therefore, a thicker film decreases $\zeta_{\mathrm{i}}$, and thus reduces the film's stiffness. This reduced stiffness leads to a smoother deceleration and longer contact time during impacts, and eventually decreases the impact force generated when a device collides with the shock stop [1-3]. But the maximum film thickness is limited by fabrication challenges and limitations.

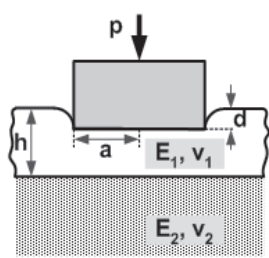

(a)

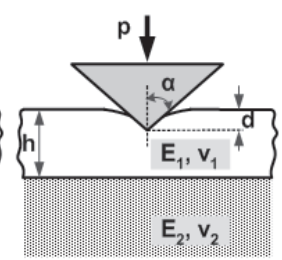

(b)

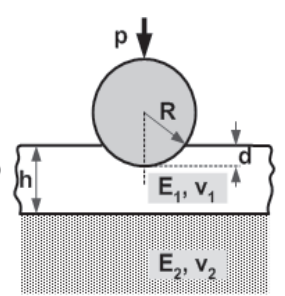

(c)
Figure 2. A thin film layer on a semi-infinite substrate indented by (a) a rigid flat-ended indenter, (b) a conical indenter, or (c) a spherical indenter [4].

Figure 3 shows the elastic energy vs. deflection of the thinfilm (Parylene film. $\zeta_{\mathrm{i}}$ is calculated to be $20 \mathrm{GPa}$ from Parylene's $\mathrm{E}_{\mathrm{i}}=3 \mathrm{GPa}, \mathrm{v}_{\mathrm{i}}=0.4$ [8].) for a device mass that has one bumper (shaped like the indenters in Figure 2) of different shapes. Also, the energy produced by a $1000 \mathrm{~g}$ shock applied to a device mass $(0.97 \mathrm{mg})$ is shown as the solid line (labeled as threshold). When the device impacts the thin film, it continues to move until the energy applied by the shock is completely converted to elastic energy stored in the thin film. The intersection points of the elastic energy plots of various indenters with the threshold shock energy show the minimum film deflections, and therefore the minimum film thickness, needed to absorb this shock. Figure 4 shows the time record of (a) the displacement of a device mass and (b) the impact force involved during this movement. In these figures, a $1000 \mathrm{~g}$ shock is applied just before the mass $(0.97 \mathrm{mg})$ touches the thin film coating. From these figures, we can derive the contact time $(\mathrm{T})$, which is inversely proportional to the average impact force (Figure 4-a), and the maximum involved impact force (Figure 4-b). Clearly, the shock performance of soft coating stops depends on both the coating's material properties (material selection and thickness) and the shock bumper design (shape and dimension). Consequently, the shock stop can be readily designed to accommodate a wide range of incident shock.

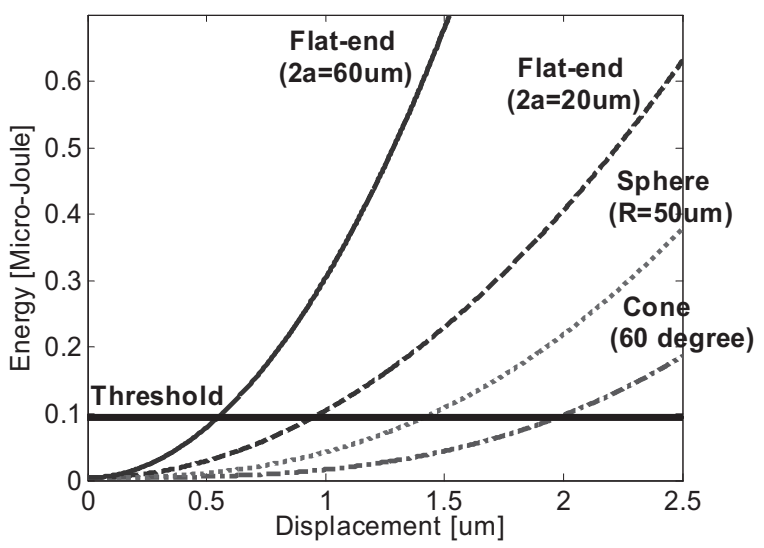

Figure 3. Elastic energy vs. deflection of a Parylene film $\left(\zeta_{i}\right.$ is assumed to be 20GPa) for a device mass that has one bumper (shaped like the indenters in Figure 2) of different shapes. The energy produced by a $1000 \mathrm{~g}$ shock applied to a device mass $(0.97 \mathrm{mg})$ is shown as the solid line (labeled as threshold).

\section{FABRICATION}

To demonstrate shock protection improvement using soft coating shock stops, we used the Silicon-On-Glass (SOG) process [3] to fabricate MEMS test structures and Parylene coated shock stops. Figure 5 shows the fabrication process flow. We first create a recess $(10 \mu \mathrm{m})$ on a Pyrex glass wafer and pattern a shield metal layer (5-a). The glass wafer is halfway diced (to form scribe lanes), and is anodically bonded to a $100 \mu \mathrm{m}$-thick, doublepolished silicon wafer. Contact pad metal is deposited and patterned (5-b), and a DRIE through wafer etch is preformed to form the device and shock stops (5-c). Finally, some samples are coated with a $3 \mu \mathrm{m}$-thick Parylene film to form soft coating shock stops (5-d), while some samples are completed without this coating to make hard shock stops.

Parylene is selected as the coating material due to its conformal coverage and room-temperature deposition $[\mathbf{9 ,} \mathbf{1 0}]$. In addition, Parylene is chemically stable [11]. Figure 6 is the SEM views of suspended microbeams covered with a $3 \mu \mathrm{m}$-thick layer of Parylene, showing the excellent step coverage of Parylene.

Figure 7 shows fabricated hard (silicon) and soft coated (Parylene) shock stops and devices. For a fair comparison, both devices are selected from the same wafer and have identical dimensions. To compensate for the decrease in gap due to Parylene deposition, the gaps of devices covered with the soft coating are made larger than the gaps of hard stop devices. Because Parylene is thin with small Young's modulus, changes in the mass and stiffness due to Parylene are ignored [12]. 
The test device consists of a silicon mass of $0.97 \mathrm{mg}(1.15 \mathrm{~mm}$ radius, $100 \mu \mathrm{m}$ thickness, (100) silicon orientation), and a support cantilever beam with a spring constant of $79 \mathrm{~N} / \mathrm{m}(50 \mu \mathrm{m}$ width, $1.85 \mathrm{~mm}$ length, and $100 \mu \mathrm{m}$ thickness). These test structures were designed to be flexible enough to touch the wall, but not to suffer fracture stress before touching the wall.

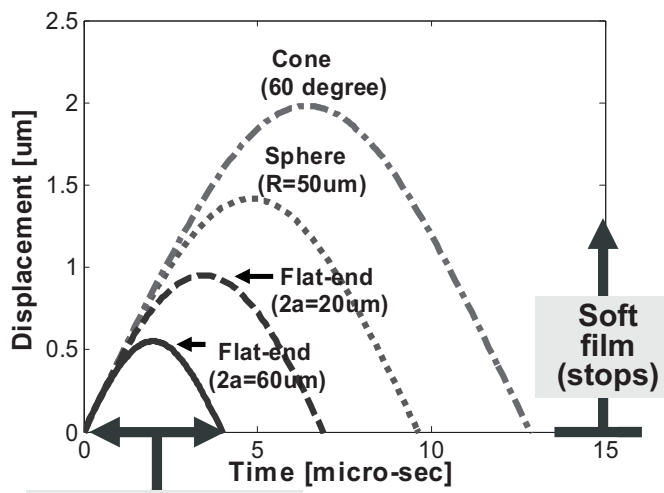

Contact time (T)

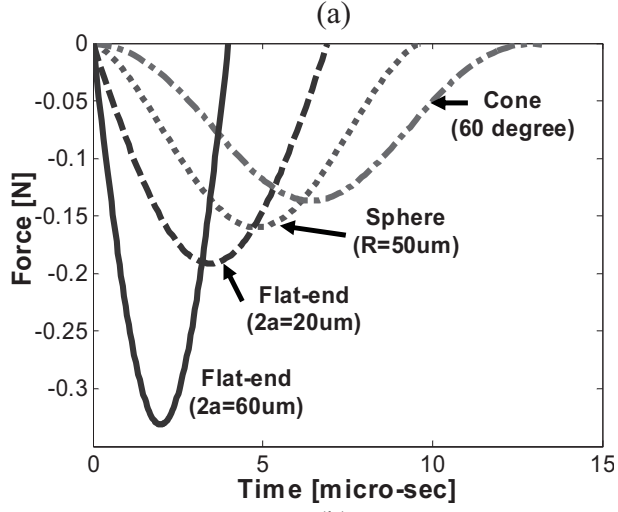

(b)

Figure 4. The time record of (a) the displacement of a device mass and (b) the involved impact force during this movement

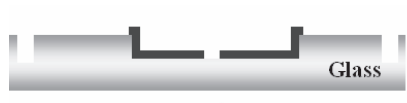

a)

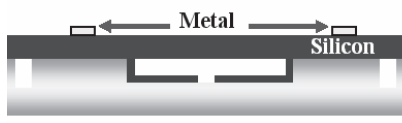

b)

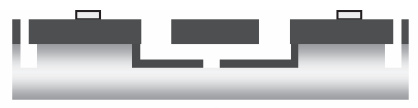

c)

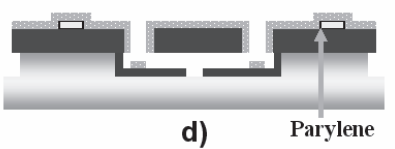

Figure 5. Fabrication process for test devices and shock stops.

\section{TEST RESULTS}

Fabricated devices were tested using a custom-built drop test setup, which can provide a controlled shock amplitude and direction.

In this drop test setup, samples are attached to a steel plate using epoxy and dropped from a known height $(\mathrm{H})$ though guides to produce directional and repeatable shocks. For fair comparison, both hard wall and soft coating samples were mounted together, as shown in Figure 7. The steel plate collides with a steel rail at the foot of the drop test track. The small contact area between the steel plate and the steel rail decreases the contact time and generates higher shock amplitude for the same drop height. This contact time is determined by applying a $5 \mathrm{~V}$ signal between the plate and the rail, and by measuring the time during which electrical contact is first achieved between the two. This critical contact time $(d T)$ determines the maximum shock amplitude applied to the test devices. This maximum shock amplitude can be estimated based on the computed velocity change during this time interval:

$$
a_{\text {shock }}=\frac{(C O R+1) \cdot \sqrt{2 \cdot 9.8 \cdot H}}{d T}
$$

While this is the shock delivered to the steel plate, we assume that the test devices experience the same shock as they are firmly attached to the plate. Also, we assume that the energy losses due to friction and the epoxy layer are negligible. Based on these assumptions and the dimensions and the size of our setup, our drop tests can produce a maximum shock of $1400 \mathrm{~g}$ when the steel plate is dropped from a height of $\sim 8$ feet.

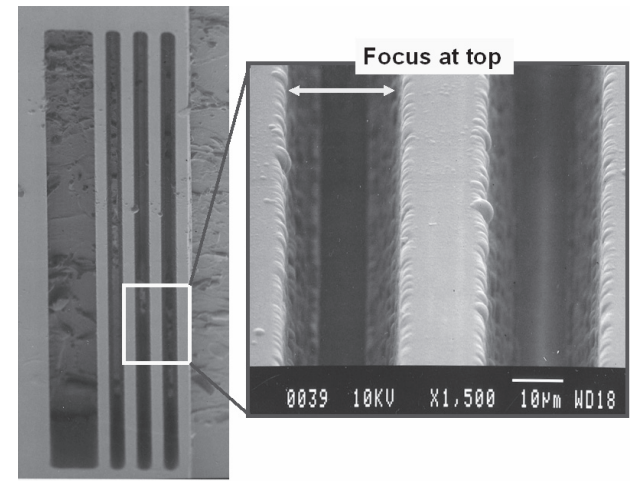

Figure 6. SEM of the top view of suspended microbeams after Parylene deposition. It shows excellent step coverage.

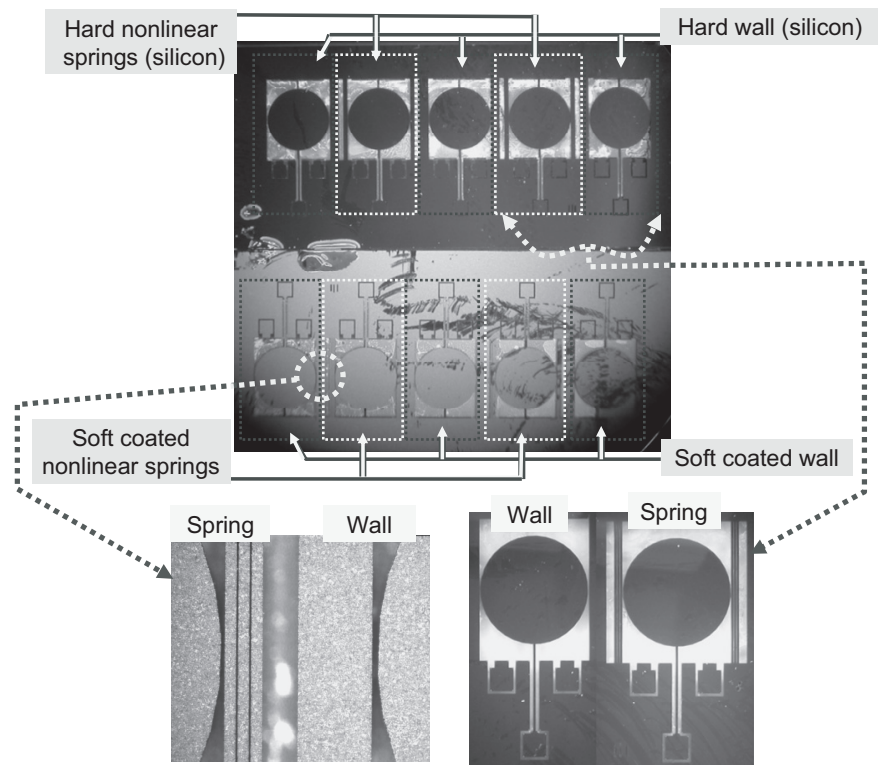

Figure 7. Top views of the fabricated hard (silicon) and soft coated (Parylene) devices. Each sample has three wall and two nonlinear spring devices. These two samples are glued together to a steel plate for testing.

Testing started by first applying a low level of shock to test devices and then proceeded to larger shocks by dropping the test devices from higher distances. Figure 8 shows a series of photographs of the test samples containing both hard wall and soft coating shock stops following each drop test. Shocks under $\sim 640 \mathrm{~g}$ produced no observable damage to either hard or soft stop devices 
(8-a). When $\sim 840 \mathrm{~g}$ was applied, one hard stop device broke (8-b). The two remaining hard stop devices were damaged after a shock of $\sim 940 \mathrm{~g}$ (8-c). But, for shocks up to $\sim 1300 \mathrm{~g}$, no damage was observed on any soft stop devices (8-d). We conducted this series of experiments four times using four different samples each containing several hard and soft shock stops, and the results are summarized in Table 1. The table clearly shows that soft coating shock stops provide superior shock protection providing a $94 \%$ survival rate, compared with $0 \%$ survival rate for hard stops for shocks of up to $1300 \mathrm{~g}$.

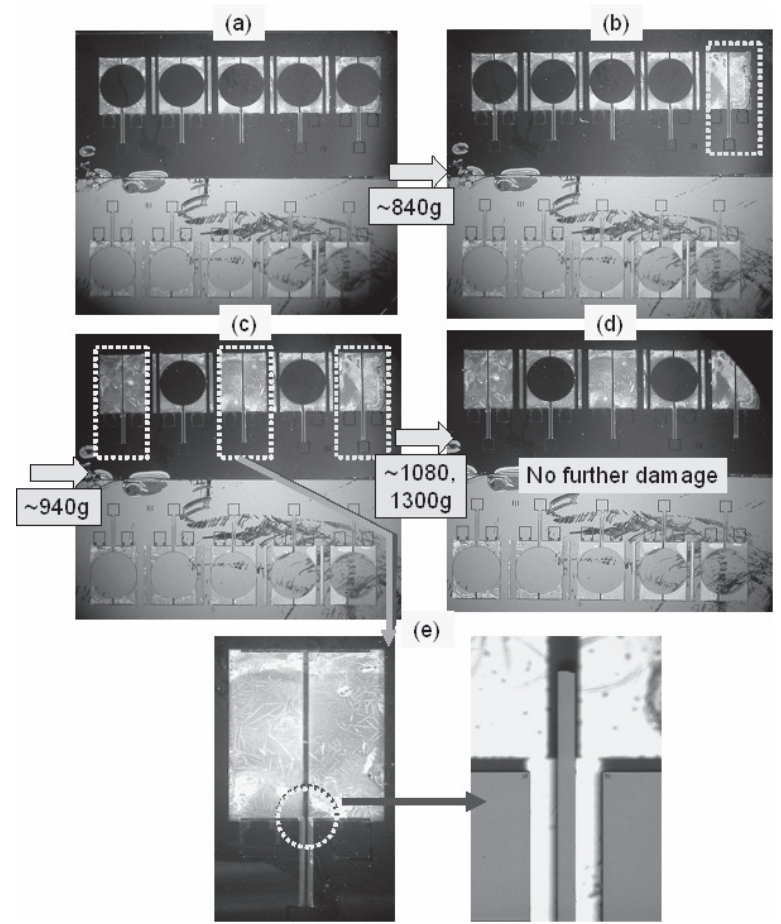

Figure 8. A series of photographs of the test samples containing both hard wall and soft coating shock stops following each drop test (up to $1300 \mathrm{~g}$ shock amplitude) for the device shown in Figure 7. All hard stops were damaged at the tip close to the device mass.

Table 1. Summary of drop test results. No hard stop devices survived, compared with 94\% of Parylene coating shock stops.

\begin{tabular}{|c|c|c|c|}
\hline \multirow{2}{*}{ Test number } & $\begin{array}{c}\text { Max. } \\
\text { applied } \\
\text { shock [g] }\end{array}$ & $\begin{array}{c}\text { Hroken / Total } \\
\text { number }\end{array}$ & $\begin{array}{c}\text { Broken /Total } \\
\text { number }\end{array}$ \\
\hline 1 & $\sim 1300$ & $\mathbf{3 / 3}$ & $\mathbf{0 / 3}$ \\
\hline 2 & $\sim 900$ & $\mathbf{3 / 3}$ & $\mathbf{0 / 3}$ \\
\hline 3 & $\sim 920$ & $\mathbf{2 / 2}$ & $\mathbf{0 / 5}$ \\
\hline 4 & $\sim 1080$ & $\mathbf{2 / 2}$ & $\mathbf{1 / 6}$ \\
\hline Summary & & $\mathbf{1 0 / 1 0}(\mathbf{1 0 0} \%)$ & $\mathbf{1 / 1 7}(\mathbf{6} \%)$ \\
\hline
\end{tabular}

Using the dimensions of our spherical device and the models in Figures 3 and 4, we derived the average impact force and the maximum impact force of both Parylene coated soft shock stops and hard silicon stops. The result shows that Parylene coated stops provide significant reduction both in average impact force $(78 \%$ reduction) and in the maximum impact force (78\% reduction), compared to hard silicon shock stops. This substantial impact force reduction considerably decreases the stress induced at the tip of the device beam [3], and thus explains why soft coating stops produce higher device survival rate in our drop test experiments. This impact force reduction is calculated using the elasticity properties of Parylene films, whereas the impulse reduction $(>40 \%)$ in $[1,2]$ derived solely from the reduced COR afforded by gold coatings.

\section{CONCLUSION}

This paper describes the modeling, fabrication and testing of soft coating shock stops. This soft coating relies on both increased surface compliance and reduced coefficient of restitution, and provides better shock protection over conventional hard wall shock stops. This shock protection is determined not only by the coating material properties but also the design of bumpers attached to a device mass. As a coating material, we selected Parylene due to its conformal coverage, room-temperature deposition, and chemical stability. Using a drop test setup, we conducted multiple shock tests, and the results demonstrate that the Parylene coating provides superior device survival rate $(94 \%)$ over hard stops $(0 \%)$. This can be explained by the fact that Parylene coating shows substantial impact force reduction compared to hard silicon stops.

\section{ACKNOWLEGEMENT}

This work is supported by DARPA's HERMIT program (contract \#: W31P4Q-04-1-R001). The authors thank Ms. A.B. Ucok \& Mr. H. Kim for their help in Parylene deposition.

\section{REFERENCES}

[1] S. W. Yoon, N. Yazdi,, N.C. Perkins, and K. Najafi, "Novel integrated shock protection for MEMS", Tech. Digest of Int. Conf. Solid-State Sensors, Actuators \& Microsystems (Transducers'05), Seoul, Korea, 396-400 (2005)

[2] S. W. Yoon, N. Yazdi, N. C. Perkins, and K. Najafi, "Micromachined integrated shock protection for MEMS", Sensors and Actuators A: Physical (2006)

[3] S.W. Yoon, N. Yazdi, J. Chae, N.C. Perkins, and K. Najafi, "Shock protection using integrated nonlinear spring shock stops," Technical Digest of IEEE Int. Conf. on Micro Electro Mechanical Systems (MEMS 2006), Istanbul, Turkey, 702-705 (2006)

[4] H. Y. Yu, S. C. Sanday and B. B. Rath, "The effect of substrate on the elastic properties of films determined by the indentation test - axisymmetric boussinesq problem", J. of the Mechanics and Physics of Solids, 38, 745-64 (1990)

[5] S. M. Han, R. Saha, and W.D. Nix, "Determining hardness of thin films in elastically mismatched film-on-substrate systems using nanoindentation”, Acta Materialia, 54, 157181 (2006)

[6] X. Chen and J.J. Vlassak, "Numerical study on the measurement of thin film mechanical properties by means of nanoindentation", J. Materials Res., 16, 2974-82 (2001)

[7] P. M. Ramsey, H. W. Chandler and T. F. Page, "Modeling the contact response of coated systems", Surface and Coatings Technology, 49, 504-9, (1991)

[8] T. Harder, T.J. Yao, Q. He, C.Y. Shih and Y.C. Tai, "Residual Stress in thin-film Parylene-C", Technical Digest IEEE Int. Conf. on Micro Electro Mechanical Systems (MEMS 2002), Las Vegas, USA, 435-438 (2002).

[9] L. Morisset, "Conformal Coating Through Gas Phase Polymerization”, OnBoard Technology, 32-34 (2003)

[10] H. Kim and K. Najafi, "Characterization of low-temperature wafer bonding using thin-film parylene", IEEE J. of Microelectromechanical Systems, 14, 1347-55 (2005)

[11] A. Campbell and J. Scialdone, "Outgassing Data for Selecting Spacecraft Materials", NASA Reference Publication 1124, Rev. 3 (1990)

[12] E. P. Popov, "Engineering mechanics of solids," PrenticeHall (1990) 